\title{
Chronic Draining Ear and Cholesteatoma Recidivism: A Retrospection from Clinical, Imaging, and Surgical Perspectives
}

\author{
Saumik Das ${ }^{1}$ (D), Mainak Dutta ${ }^{1}$ (D), Tanaya Panja ${ }^{2}$ (D), Ramanuj Sinha ${ }^{1}$ (D) \\ Original Investigation $>{ }^{1}$ Department of Otorhinolaryngology and Head-Neck Surgery, Medical College and Hospital, Kolkata, India \\ ${ }^{2}$ Department of Otorhinolaryngology and Head-Neck Surgery, Chandannagar Subdivisional Hospital, Hooghly, India
}

Abstract

ORCID IDs of the authors: S.D. 0000-0003-2353-3398; M.D. 0000-0003-3977-3230; T.P. 0000-0002-0366-1838; R.S. 0000-0001-7215-4533.

Cite this article as: Das S, Dutta M, Panja T, Sinha R. Chronic Draining Ear and Cholesteatoma Recidivism: A Retrospection from Clinical, Imaging, and Surgical

Perspectives. Turk Arch Otorhinolaryngol 2019; 57(3): 133-9.

\section{Corresponding Author:}

Mainak Dutta; duttamainak@yahoo.com

Received Date: 13.03.2019

Accepted Date: 23.06.2019

Content of this journal is licensed under a Creative Commons Attribution 4.0 International License. Available online at www.turkarchotolaryngol.net
Objective: To evaluate the reasons for persistent draining ear and cholesteatoma recidivism following canal wall down (CWD) tympanomastoidectomy by studying the sensitivity of high-resolution computed tomography (HRCT) scanning in different potential etiologies, corroborating through appropriate surgical intervention, and thereby, to suggest proper preventive measures.

Methods: In this observational study, 32 chronic, refractory draining ears were subjected to revision surgery following a radical or a modified radical mastoidectomy. Besides disease (cholesteatoma/granulations) eradication, pitfalls of the primary surgeries were addressed. Data were interpreted for studying the epidemiologic profile, the clinical presentation at recurrence, the type of primary surgery, the sites of recidivism, the probable causes, and the best possible management at revision.

Results: Of the 32 patients/ears, 23 had residual/ recurrent cholesteatoma. Major reasons were inade- quate disease clearance, contracted/inadequate conchomeatoplasty, no cavity obliteration, and inappropriate bone work. HRCT predicted persistent bridge and lateral semicircular canal dehiscence with 100\%, and ossicular integrity and bony overhang with $>80 \%$ sensitivity. Sinus tympani and oval window niche were the commonest sites of recurrence. At revision, $\mathrm{radical} /$ modified radical mastoidectomies were associated with cavity obliteration and appropriate revision of conchomeatoplasty in 28 patients.

Conclusion: Recurrence of cholesteatoma/granulations is an important cause for chronic drainage from post-CWD cavities. Revision surgery explores the surgical pitfalls, and ensures clearance of disease from hidden areas, adequate bone work, and optimum conchomeatoplasty following cavity obliteration to provide a safe, dry ear with hearing improvement whenever feasible.

Keywords: Chronic draining ear, cholesteatoma, surgery, recurrence

\section{Introduction}

Cholesteatoma is notorious for causing complications due to its bone-eroding nature. Its management is essentially surgical, mostly a canal wall down (CWD) tympanomastoid surgery in advanced conditions (1). One of the greatest challenges after a successful management of squamous chronic otitis media (COM) is to combat a chronic draining ear and cholesteatoma recidivism (2). The latter can result from either residual or recurrent disease, following canal wall up (CWU) or CWD procedures, respectively (3). In spite of being in the antibiotic era and equipped with better visual aids and surgical instruments, even the most recent literature document recurrences that range from 13.2 to $22.1 \%$ in CWD surgeries (3). A chronic wet ear and cholesteatoma recidivism also have considerable bearing on the personal, emotional and social life of the patients, adversely affecting the quality of life factors. Furthermore, such events necessitate revision tympanomastoid surgery and thereby defeat the very purpose of the primary surgery, that of clearing disease from the tympanic cavity and all accessible mastoid air cells to achieve a safe and dry ear and creating a manageable compartment to minimize post-operative cavity problems.

Although contemporary literature traditionally highlights the rationale, the necessity and the technical details of a primary surgical procedure 
Das et al.

and emphasizes its outcome, there is a dearth of studies that deal with revision tympanomastoid surgeries, their indications, per-operative findings, and most importantly, with the identification of the factors responsible for revision surgery. In this context, high resolution computed tomography (HRCT) scanning of the temporal bones provides a blueprint of the possible etiologies that could have led to recidivism, that guides the otologist to plan the surgery accordingly. This study emphasizes the importance of interpreting the sensitivity of HRCT scanning by observing how the per-operative findings corroborate those of the imaging suggestions. It needs to be acknowledged that the available evidence-based information mostly originate from the western literature, and that cholesteatoma and its inevitable fallout, including its surgical management and subsequent complications, are distinct in developing and poorer nations due to the differences in socio-economic and demographic profile (4). The shift in the contemporary surgical practice towards more conservative and canal wall reconstructive (CWR) approaches-a vision perhaps more suited for limited disease as usually encountered in the developed nations might be another factor for the scarcity of literature dealing with the so-termed pitfalls of classic CWD approaches and the ways to manage them (4). Realizing the need of representative and reliable information from a geographic domain where cholesteatoma surgery often needs to be extensive as well as aggressive, the present study attempts to re-evaluate the causes of persistent draining ear and cholesteatoma recidivism following CWD tympanomastoid surgery by pre- and per-operative identification of surgical pitfalls, and suggests appropriate measures to prevent them.

\section{Methods}

This observational study was conducted in a tertiary-care teaching hospital from March 2016 to February 2018. In this period, 32 patients with persistent draining of the operated tympanomastoid cavity that refused to subside after optimum medical management (regular ear mopping, suction/clearance under microscope with/without the aid of otoendoscope, oral antibiotics, topical steroid-antibiotic ear drops, and diluted acetic acid wash) for a minimum of six months following their previous CWD procedure (radical/modified radical mastoidectomy) were subjected to revision surgery. Their primary surgeries were done either in the same or in an external clinic; accordingly, the patients were followed-up either starting immediately after the primary surgery, or when referred from other hospitals. The identity of the surgeons performing the primary surgeries were kept undisclosed to prevent any bias inadvertently creeping into the study.

All patients were operated on one ear only, hence 32 ears of 32 patients were included in the study. Each patient was thoroughly evaluated based on their earlier surgical records, relevant history, recent HRCT scanning of temporal bones, examination under microscope with or without otoendoscopy, and hearing assessment with pure tone audiometry (PTA) considering parameters like pure tone average (PTAv) and air-bone gap (ABG). Patients who had CWR surgeries (e.g., atticotomy with canal and scutum reconstruction), and those with clinico-radio- logic evidence of intracranial complications were excluded. All revision surgeries were performed by a single group of surgeons following a standardized protocol.

The revision surgeries were carried out with the aim of eradicating the causes of draining ear, including recurrent cholesteatoma, and addressing the pitfalls of the primary surgeries, such as adequate lowering of facial ridge, complete removal of facial bridge, saucerization of the tympanomastoid cavity, truncating the mastoid tip region, cavity obliteration, and revising the conchomeatoplasty as needed following cavity obliteration. Hearing reconstruction was performed under suitable conditions. The pre- and per-operative data were collected and interpreted for studying the epidemiologic profile of the patients, clinical presentation at recurrence, types of primary surgery, sites of recidivism, probable causes (including identifying the pitfalls of primary surgery as stated earlier), and the best management that could be provided in secondary (revision) surgery. Besides the operating microscope (Carl Zeiss Meditec AG, Germany; Model: OPMI 1FC), otoendoscopes (Karl Storz SE and Co. KG, Tuttlingen, Germany) of $0^{\circ}$ and $30^{\circ}$ were used when required, especially while addressing difficult-to-reach areas like the sinus tympani and anterior epitympanic/supratubal recesses.

Informed consent was obtained in writing from each patient. The study received due clearance from the Ethical Committee of Medical College and Hospital, Kolkata. A level of evidence of $2 \mathrm{~b}$ (according to the Oxford Center of Evidence-Based Medicine) (5) has been assigned to this study.

\section{Statistical analysis}

Data were entered in Microsoft Excel 2016 software (Microsoft Corporation, Redmond, Washington, USA) and analyzed using standard statistical methods. Calculations were done using Statistical Package for the Social Sciences software version 22 (IBM Corp.; Armonk, NY, USA) using standard formulae and expressions.

\section{Results}

The study population consisted of 14 men and 18 women aged 10 to 55 years (mean age: 33.27 years). The chief presenting complaints included persistent ear discharge in all the patients, along with hearing impairment ( 84\%) (Table 1). Vertigo ( 16\%) and facial paresis $(\sim 9 \%)$ were present in a minority of the patients. PTA done during preparation for the revision surgery showed

Table 1. Distribution of patients according to presenting complaints

\begin{tabular}{l|c|c}
\hline Presenting Complaint & $\begin{array}{c}\text { Number of Patients } \\
(\mathbf{n}=32)\end{array}$ & $\%$ \\
\hline Persistent ear discharge & 32 & 100 \\
\hline Hearing Impairment & 27 & 84.38 \\
\hline Vertigo & 5 & 15.63 \\
\hline $\begin{array}{l}\text { Facial paresis (one patient had HB* } \\
\text { grade II and the other two }\end{array}$ & 3 & 9.38 \\
$\begin{array}{l}\text { Grade IV paresis) } \\
\text { *House-Brackmann }\end{array}$ & & \\
\hline
\end{tabular}


conductive impairment in 19 and mixed impairment in 13 patients. The mean pre-operative PTAv and ABG were $59 \mathrm{~dB}$ and $38.7 \mathrm{~dB}$, respectively.

The primary surgeries included modified radical mastoidectomy in $24(75 \%)$ and radical mastoidectomy in $8(25 \%)$ patients (Table 2). The mean interval between the primary and the revision surgeries was $3.62 \pm 1.65$ years.

Findings at revision surgery were corroborated with those in the HRCT of temporal bones prior to and during surgery (Table 3 ). It was noted that the HRCT findings were $100 \%$ sensitive in predicting inadequately removed facial bridge and dehiscence of the lateral semicircular canal and were $>80 \%$ sensitive in determining the presence of ossicles and bony overhang. It was found to be less satisfactory for predicting breaches in the tegmen and sinus plates, presence of high facial ridge and Fallopian canal dehiscence.

Combination of pre-operative examination under microscope/ otoendoscope and per-operative findings revealed the stigmata of previous surgery that could explain the presenting complaints of the patients (Table 4). Of the 32 patients subjected to revision surgery, 23 were found to have residual/recurrent cholesteatoma. The major cause of surgical failure was deemed to be inadequate disease clearance that led to granulations and recurrence of cholesteatoma (Table 4). The other important causes included contracted/inadequate conchomeatoplasty and improper bone work leading to inadequate saucerization, high facial ridge, inadequately removed facial bridge, and sump effect. Among the patients with cholesteatoma recidivism, sinus tympani and oval window niche were the commonest sites of recurrence $(\sim 87 \%$ and $\sim 65 \%$, respectively), followed by tip cells, supratubal recess, residual cells in the mastoid cavity, anterior epitympanic and facial recesses, in order (Table 5). Overall, among the 32 subjects, residual ossicles with Austin Kartush type $\mathrm{C}$ status (M-S+) were detected in 18 patients, while no stapes could be visualized in the rest (Austin Kartush type D; M-S-). Residual partial/total ossicular reconstruction prostheses were found in 11 patients. In none of the ear cavities obliteration was attempted.

An overview of the surgical procedures at revision has been summarized in Table 6. Radical mastoidectomy had to be performed in two patients with pre-operative mixed hearing impairment with $\mathrm{ABG}<20 \mathrm{~dB}$ (including one with a facial nerve decompression procedure), and in another two patients where the oval window niche could not be completely cleared off from the granulations. In the latter group, a second-stage surgery for ossiculoplasty was planned. Facial nerve decompression was performed in two patients having House-Brackmann grade IV facial paresis. The mastoid cavity was obliterated in 28 patients with suitable materials in variable combinations that included temporalis muscle pedicle flap, bone dust and cartilage pieces obtained from the residual concha and/or tragus. In the rest, the mastoid cavities were

Table 2. Distribution of patients according to type of primary surgery

\begin{tabular}{l|l|c|c}
\hline \multirow{2}{*}{ Type of Surgery } & & $\begin{array}{c}\text { Number of Patients } \\
(\mathbf{n}=32)\end{array}$ & $\begin{array}{c}\text { Total Number of } \\
\text { Patients }(\%)\end{array}$ \\
\hline Modified Radical Mastoidectomy* & Type III Minor Columella Tympanoplasty with PORP & 10 & $24(75)$ \\
\cline { 2 - 3 } & Type III Major Columella Tympanoplasty with TORP & 5 \\
\hline Radical Mastoidectomy & Type III Stapes Columella & 5 & $8(25)$ \\
\hline
\end{tabular}

PORP/TORP: Partial/Total Ossicular Replacement Prosthesis

*Disease clearance and ossicular reconstructions were performed in the same session

Table 3. Corroboration of HRCT of temporal bone with per-operative findings $(n=32)$

\begin{tabular}{l|c|c|c}
\hline Parameters & $\begin{array}{c}\text { HRCT findings } \\
\text { (Number of Patients/ears) }\end{array}$ & $\begin{array}{c}\text { Per-operative findings } \\
\text { (Number of Patients/ears) }\end{array}$ & $\begin{array}{c}\text { Sensitivity } \\
\text { (HRCT) }\end{array}$ \\
\hline Presence of ossicles & 15 & 18 & $83.33 \%$ \\
\hline Bony overhang & 19 & 24 & $82.76 \%$ \\
\hline High facial ridge & 15 & 12 & $77.78 \%$ \\
\hline Inadequately removed facial bridge & 12 & 8 & $100 \%$ \\
\hline Facial canal dehiscence* & 5 & 3 & $72.73 \%$ \\
\hline LSCC dehiscence* & 3 & 21 & $100 \%$ \\
\hline Tegmen dehiscence** & 13 & 9 & $72.41 \%$ \\
\hline Sinus plate dehiscence** & 6 & & $75 \%$ \\
\hline
\end{tabular}

*Either congenital or due to erosion by primary or recurrent cholesteatoma

**Due to erosion by cholesteatoma

*** Either due to erosion by primary or recurrent cholesteatoma, or iatrogenic

LSCC: lateral semicircular canal; HRCT: high resolution computed tomography 
Das et al.

either dry and contracted enough, or harbored discrete areas of cell-pockets with granulations/edematous mucosa (in two pediatric patients), to preclude an attempt at obliteration. The need for refashioning conchomeatoplasty was subsequently analyzed according to the extent of cavity obliteration.

Table 4. Revelations at revision surgery explaining the chronic draining ear following primary CWD surgery

\begin{tabular}{l|c|c}
\hline Findings & $\begin{array}{c}\text { Number of } \\
\text { Patients/Ears } \\
(\mathbf{n}=32)\end{array}$ & $\%$ \\
\hline Recurrence of cholesteatoma & 23 & 71.86 \\
\hline Granulations & 26 & 81.25 \\
\hline High facial ridge & 21 & 65.63 \\
\hline Inadequately removed facial bridge & 12 & 37.5 \\
\hline Bony overhang/inadequate saucerization & 24 & 75 \\
\hline Sump effect & 13 & 40.63 \\
\hline $\begin{array}{l}\text { Inadequate/contracted/cicatrized } \\
\text { conchomeatoplasty }\end{array}$ & 27 & 84.38 \\
\hline
\end{tabular}

Table 5. Distribution of patients according to the site of cholesteatoma recurrence

\begin{tabular}{l|c|c}
\hline Site of Residual Cholesteatoma* & $\begin{array}{c}\text { Number of Cases } \\
(\mathbf{n}=23)\end{array}$ & $\%$ \\
\hline Tip cells & 13 & 56.52 \\
\hline Sinodural angle Cells & 7 & 30.43 \\
\hline Facial recess & 10 & 43.48 \\
\hline Sinus tympani & 20 & 86.96 \\
\hline Round window niche & 8 & 34.78 \\
\hline Oval window niche & 15 & 65.22 \\
\hline Protympanum & 6 & 26.1 \\
\hline Supratubal recess & 12 & 52.17 \\
\hline Anterior epitympanic recess & 10 & 43.48 \\
\hline $\begin{array}{l}\text { Posterior epitympanic area } \\
\text { Perilabyrinthine cells }\end{array}$ & 5 & 21.74 \\
\hline $\begin{array}{l}\text { Perifacial and retrofacial cells } \\
\text { Residual cells in the mastoid cavity }\end{array}$ & 12 & 30.43 \\
\hline $\begin{array}{l}\text { *Anatomic terms like posterior epitympanum/epitympanic recess (replaced here by } \\
\text { posterior epitympanic area), aditus, hypotympanum, etc. are not used here because these } \\
\text { areas are ill-defined or non-existent in a CWD radical/modified radical mastoidectomy }\end{array}$ \\
\hline
\end{tabular}

\section{Discussion}

Recent data from the World Health Organization in 2004 revealed the huge burden of morbidity due to COM, overall amounting to a loss of $>2$ million DALYs (Disability Adjusted Life Years) worldwide, $>90 \%$ of which is in South-East Asia, Western Pacific region and Africa (6). Surgery is the only effective treatment for cholesteatoma with the primary aim of eradicating the disease (1). Failure to achieve this might lead to recurrent/residual cholesteatoma with associated infective and ineffective reparative process resulting in granulations. These result in chronic discharging ear and hearing impairment necessitating revision surgery.

Even with meticulous history-taking, clinical examination and examination under microscope/otoendoscope, it is difficult to distinguish at revision surgery the differences between the two components of cholesteatoma recidivism, i.e., residual and recurrent disease. Although presenting with similar symptoms, they differ in pathogenesis. A canal wall up (CWU) tympanomastoid surgery is more vulnerable for residual cholesteatoma, unlike a CWD procedure where recurrence complicates the post-operative period $(3,7)$. Among its many forms, a residual/recurrent cholesteatoma might develop as a whitish keratin pearl displacing the tympanic membrane or present within the mastoid cavity (7). However, in the present era of CWR procedures, and especially when the primary disease is mostly of limited extent within the urban population with improved awareness and hygiene, the relationship between a CWD and a CWU procedure with the nature of cholesteatoma recurrence appears blurred (4). Nevertheless, in the present study, we have ignored CWR procedures and considered only CWD as the primary surgery (which implies that the cholesteatoma was extensive enough to warrant a CWD procedure as the primary surgery). In the process, we have not further attempted to differentiate residual from recurrent cholesteatoma/granulations and have termed such disease pathology uniformly as recurrence.

Since the tympanomastoid cavities were not obliterated in the primary surgeries, the major complaints of the patients were mostly due to chronic inflammation of a large cavity epithelial lining. This was complicated by cholesteatoma recurrence and its sequelae, like facial paresis. In fact, the commonest reason for frequent visits to otolaryngologists following CWD procedures is intractable ear discharge due to keratin accumulations,

Table 6. Distribution of patients according to the broad surgical procedures at revision

Surgical Procedures

Modified radical mastoidectomy
Type III Tympanoplasty with PORP (Minor Columella)

Type III Tympanoplasty with TORP (Major Columella)

Type III Tympanoplasty with Stapes Columella

Type IV Tympanoplasty
Number of Patients (\%) ( $\mathrm{n}=32)$

$10(31.25)$

$9(28.13)$

7 (21.88)

2 (6.25)

$4(12.5)$

$2(6.25)$

$28(87.5)$

Facial nerve decompression

Cavity obliteration 
and granulation tissues that require repeated cavity care (8). The average follow-up period in such conditions might even be up to five years (9).Most importantly, the need for revision surgery becomes evident when such a chronic draining cavity refuses to heal in spite of optimal medical management for a given period (an arbitrary time period of a minimum of six months was considered in this study).

The chief aim of revision surgery is to eradicate the disease, and also to evaluate the reasons for recidivism-the probable pitfalls of earlier surgical attempts—and addressing them to prevent further recurrence. Evaluation of HRCT of temporal bones constitutes an important step in getting prepared for revision surgery. It is a challenging task for the otologist, given the loss and distortion of landmarks owing to the primary surgery and disease recurrence. A pre-operative HRCT not only determines the extent of the disease and excludes any asymptomatic complication, it is also necessary for studying the basic anatomy, to detect loss of surgical landmarks, and to rule out congenital anomalies (10). Because of the known limitation of CT scan in not being able to determine the nature of the soft tissue in the tympanomastoid cavity (cholesteatoma/granulations/mucosal or epithelial edema/debris) (11), we have not looked for the same in our study on imaging (Table 3). Nevertheless, it provided invaluable information regarding the stigmata of earlier surgery and the present disease burden, so that the revision surgery could be accordingly planned. In the present study, HRCT of temporal bones was useful in detecting inadequately removed facial bridge, dehiscence of the lateral semicircular canal, ossicular status and bony overhang, while it was relatively less informative in predicting high facial ridge and dehiscence of the tegmen, the sigmoid sinus plate and the Fallopian canal. Our experience has been shared by authors like Thukral et al. (12) who reported a high sensitivity and specificity of HRCT in identifying lateral semicircular canal dehiscence and high specificity in detecting Fallopian canal dehiscence.

The definitive proof of residual/recurrent cholesteatoma and understanding the pathophysiology of chronic draining ear could be realized only at surgical exploration, which confirms the clinical suspicion and radiologic suggestions. Our study revealed, in order, sinus tympani, oval window niche, tip cells, supratubal recess, residual mastoid cells, facial recess and anterior epitympanic space, among others, as the areas for cholesteatoma recurrence. These findings, and also those in chronic draining ears not due to cholesteatoma, would essentially vary with studies depending upon interlinked, multifactorial etiologies like the strength of study population, extent of the primary disease, type and adequacy of the primary surgery performed, and the socio-economic condition of the patient. Thus, it is the common observation that the most likely places for intractable diseases include anterior epitympanic space, hypotympanum and sinus tympani (10) along with sinodural angle, facial recess, oval window and tip cells. However, Megerian et al. (13) in their study showed that the areas with persistent disease included sinodural angle (92\%), tegmen cells (88\%) and tip cells (62\%), and implicated incomplete lowering of the facial ridge (94\%) and inadequate conchomeatoplasty (60\%) as the primary reasons. Likewise, in a study by Faramarzi et al. (14), the commonest site of recrudescence was sinodural angle cells $(\sim 28 \%)$, and the most important mechanical cause of failure was high facial ridge (53\%). As an important observation in our series, the reasons for chronic draining ears were not only cholesteatoma recrudescence but also persistent granulations, and the two often co-existed (Table 4). The other factors implicated were compromised conchomeatoplasty (inadequate/contracted/cicatrized), bony overhang or inadequate saucerization, and high facial ridge.

An irregular tympanomastoid cavity with nooks and crevices hampers epithelialization by natural migration of squamous epithelium from the external auditory canal and the outer layer of the tympanic membrane, and results in the accumulation of redundant epithelium, promotes a faulty reparative process forming granulation tissue, invites infection in the process and leads to a chronic draining ear (15). There are six methods of creating a manageable cavity in a CWD surgery: adequate saucerization by removing the bony buttresses and overhangs, complete removal of the bridge, adequate lowering of facial ridge, amputation of the mastoid tip, cavity obliteration, and creating a conchomeatoplasty as needed following sufficient cavity obliteration. Our present effort focused on proper bone work that followed these basic principles. Wormald and Nilssen (16) found that in dry cavities, the height of the facial ridge, aptly termed as the "beginner's hump"-an area bounded by bony horizontal semicircular canal above, tympanomastoid suture in the posterior meatal wall, and digastric ridge in the mastoid tip belowwas on average $3 \mathrm{~mm}$ lower than that in the wet cavities $(17,18)$. In the revision set-up, we lowered the facial ridge adequately under high magnification, using ample irrigation, up to the level of lateral semicircular canal, until the white nerve sheath with delicate vascularity could be identified through a thin plate of the Fallopian canal bone. On the other hand, residual tip cells and high facial ridge were the main causes of sump effect, i.e., accumulation of debris in the dependent part of the mastoid resulting in persistent ear drainage. After amputation of the mastoid tip area, the sternocleidomastoid muscle would collapse medially to obliterate this potential space, which can reduce the size of the tympanomastoid cavity by as much as $50 \%(15,19)$. In the postoperative period, soft tissues collapsed medially over the smooth, shallow mastoid bowl and ensured proper epithelialization, resulting in a relatively smaller tympanomastoid cavity.

Even after taking care of the surgical principles to ensure a manageable cavity in the postoperative period, the need for cavity obliteration cannot be overlooked. A properly obliterated mastoid cavity would minimize the overall tympanomastoid volume, cover the raw bone, and thus help in epithelialization and reduce doctor-dependence in the follow-up period. Among the many techniques, use of vascularized pedicle grafts (Palva flap, sternocleidomastoid flap, superior temporalis flap), fascia/ skin grafts, bone dust, pieces of conchal cartilage, and inorganic bone substitutes (hydroxyapatite or bioglass) are in vogue (15), although the surgeon is free to improvise his or her techniques and prefer an individual choice once the basic principles of 
tympanomastoid surgery are adhered with (20). Our institute is vigorously supporting the routine practice of cavity obliteration in CWD mastoidectomies where there is no attempt at CWR, and the agents most widely used here include cartilage pieces, bone dust (or combination thereof), and temporalis muscle flap. These procedures are often complemented by the resultant shrinkage of the mastoid cavity following amputation of the mastoid tip region (as discussed earlier) in situations where the otherwise would have led to sump effect. Another benefit of a properly obliterated cavity is a correspondingly smaller conchomeatoplasty. Although difficult to define, an "adequate" conchomeatoplasty traditionally has been considered to allow, after proper mopping, unaided visualization of the entire tympanomastoid cavity at a time, because the effective diameter of the external auditory canal is made proportional to the tympanomastoid cavity. With proper cavity obliteration, the effective size of conchomeatoplasty can be reduced considerably, thereby avoiding disfigurement and scar. This is especially vital in revision surgeries, and in patients prone to cicatrization where the scope for refashioning a contracted port is reduced. Indeed, the extent of conchomeatoplasty should be looked upon as the function of the adequacy of cavity obliteration. In our series of revision surgery, $~ 88 \%$ ears underwent cavity obliteration, and the need and the extent of refashioning the conchomeatoplasty procedures were also re-assessed accordingly.

There are some inherent limitations in the present study. A larger sample size would have provided a more representative picture of the indications and procedures of revision tympanomastoid surgery and help us understand the pathophysiology of a chronic draining ear. However, considering the huge volume of patients undergoing primary surgery for squamous COM in this tertiary care teaching institute, the proportion of ears requiring revision surgery in this two-year-period might be acceptable, especially when many of these patients were referrals with their primary surgeries performed elsewhere. That the majority of the patients continue to have favorable outcomes following primary CWD surgeries in our institute might speak of a generalized good surgical practice, and re-establishes the notion: the most important factor for the failure of CWD surgery is related to poor surgical technique, not the type of surgery performed. Another major area discussed earlier is the realization of the importance to routinely adopt suitable obliteration techniques to prevent problems associated with large tympanomastoid cavities. The patients included in this study were not subjected to cavity obliteration in their primary surgeries, and this might have contributed to the disease burden. The recent change in our current surgical practice should address this aspect satisfactorily. Finally, the context of the present study falls short in furthering our understanding of the newer trend in otology practice, that of conservative or CWR techniques, because it has dealt with the conventional CWD (radical/modified radical) mastoidectomies. Although it is evident that the study-setting adopted here precludes CWR techniques and therefore the omission cannot be its true limitation, it remains to be seen how recurrence of squamous disease would behave in CWR procedures, so that our surgical skills and understanding get further refined and need-based.
Evidently, revision tympanomastoid surgery is a challenging procedure because it has to address distorted anatomic landmarks and disease recrudescence and requires a thorough knowledge of the three-dimensional anatomy of the middle ear cleft. But its most significant implication, assisted by the corroboration with pre-operative HRCT scan, lies in the fact that it helps the surgeons to realize the faults in the primary surgery. This further acts as a caveat in preventing such recurrences in future and improvising means to optimize the favorable outcomes of the surgery, like routine practice of cavity obliteration. Revision surgery following a CWD primary surgery aims at meticulous clearance of the recurrent disease (granulations/cholesteatoma), adequate bone work, and performing appropriate conchomeatoplasty as needed following cavity obliteration, in order to provide a safe and dry ear with hearing improvement whenever feasible. The present study has dealt with the experience from a tertiary-care teaching institute with 32 ears with disease recurrence following radical and modified radical mastoidectomies and has looked for the possible causes of recidivism and described how they can be managed at revision.

Ethics Committee Approval: Ethics committee approval was received for this study from the Ethics Committee of Medical College and Hospital.

Informed Consent: Written informed consent was obtained from the patients who participated in this study.

Peer-review: Externally peer-reviewed.

Author Contributions: Concept - R.S., S.D., T.P.; Design - M.D., T.P.; Supervision - M.D., S.D., R.S.; Data Collection and/or Processing T.P., M.D.; Analysis and/or Interpretation - M.D., T.P., S.D.; Literature Search - M.D., T.P.; Writing - T.P., M.D.; Critical Reviews - M.D., S.D.

Conflict of Interest: The authors have no conflicts of interest to declare.

Financial Disclosure: The authors declared that this study has received no financial support.

\section{References}

1. Browning GG, Merchant SN, Kelly G, Swan IR, Canter R, Mckerrow W. Chronic Otitis Media. Gleeson M, Browning GG, Burton MJ, Clarke R, Hibbert J, Jones NS, et al., editors. ScottBrown's Otoloayngology, Head and Neck Surgery. 7th ed. London: Hodder Arnold; 2008. p. 3395-445. [CrossRef]

2. Olszewska E, Rutkowska J, Özgirgin N. Consensus-based recommendations on the definition and classification of cholesteatoma. J Int Adv Otol 2015; 11: 81-7. [CrossRef]

3. Kerckhoffs KG, Kommer MB, van Strien TH, Visscher SJ, Bruijnzeel H, Smit AL, et al. The disease recurrence rate after the canal wall up or canal wall down technique in adults. Laryngoscope 2016; 126: 980-7. [CrossRef]

4. Dutta M. Canal wall up versus canal wall down mastoidectomy: Considerations for the new-age otologists. J Int Adv Otol 2018; 14: 506-8. [CrossRef]

5. Oxford Centre for Evidence-based Medicine - Levels of Evidence (March 2009). Available at: https://www.cebm. net/2009/06/oxford-centre-evidence-based-medicine-levels-evidence-march-2009/ (accessed: August 23, 2018). 
6. Acuin J. Global burden of disease due to chronic suppurative otitis media: disease, deafness, deaths and DALYs. In: Chronic suppurative otitis media: burden of illness and management options. World Health Organization, Geneva, Switzerland. 2004. p. 23.

7. Parisier SC, Fayad JN. Revision surgery for cholesteatoma or chronic otitis media. Edelstein DR, Kraus D, Pastorek NJ, Selesnick SH, Ward RF, editors. Revision Surgery in Otorhinolaryngology. New York: Thieme Medical Publishers; 2009. p. 81-8.

8. Khalil HS, Windle-Taylor PC. Canal wall down mastoidectomy: A long term commitment to the outpatients? BMC Ear, Nose Throat Disord 2003; 3. Available from: URL: https://doi. org/10.1186/1472-6815-3-1 [CrossRef]

9. Sadé J. Surgical planning of the treatment of cholesteatoma and postoperative follow-up. Ann Otol Rhinol Laryngol 2000; 109: 372-6. [CrossRef]

10. Yates PD, Flood LM, Banerjee A, Clifford K. CT scanning of middle ear cholesteatoma: what does the surgeon want to know? Br J Radiol 2002; 75: 847-52. [CrossRef]

11. Banerjee A, Flood LM, Yates P, Clifford K. Computed tomography in suppurative ear disease: does it influence management? J Laryngol Otol 2003; 117: 454-8. [CrossRef]

12. Thukral CL, Singh A, Singh S, Sood AS, Singh K. Role of high resolution computed tomography in evaluation of pathologies of temporal bone. J Clin Diagn Res 2015; 9: TC07-10. [CrossRef]
13. Megerian CA, Cosenza MJ, Meyer SE. Revision tympanomastoid surgery. Ear Nose Throat J 2002; 81: 718-20. [CrossRef]

14. Faramarzi A, Motasaddi-Zarandy M, Khorsandi MT. Intraoperative findings in revision chronic otitis media surgery. Arch Iran Med 2008; 11: 196-9.

15. Robinson J. Surgery of chronic suppurative otitis media. Ludman H, Wright T, editors. Diseases of the ear. 6th ed. New Delhi: Jaypee Brothers Medical Publishers (P) Ltd; 2006. p.41628.

16. Wormald PJ, Nilssen EL. The facial ridge and the dscharging mastoid cavity. Laryngoscope 1998; 108: 92-6. [CrossRef]

17. Kriskovich M, Shelton C. Surgical treatment of chronic otitis media and cholesteatoma. Canalis RF, Lambert PR, editors. The Ear: Comprehensive Otology. Philadelphia: Lippincott Williams and Wilkins; 2000. p.447-65.

18. Kveton JF. Open cavity mastoid operations. Gulya AJ, Minor LB, Poe DS, editors. Glasscock- Shambaugh Surgery of the Ear. 6th ed. Shelton: People's Medical Publishing House; 2010. p.515-27.

19. Quaranta A, Cassano P, Carbonara G. Cholesteatoma surgery: Open vs closed tympanoplasty. Am J Otol 1988; 9: 229-31.

20. Chan CY, Chan YM. Mastoid obliteration and reconstruction: A review of Ttchniques and results. Proceedings of Singapore Healthcare 2012; 21: 23-9. [CrossRef] 